\title{
Pulmonary and Physical Rehabilitation in Critically III Patients
}

\author{
Myung Hun Jang', Myung-Jun Shin ${ }^{1,2}$, Yong Beom Shin ${ }^{1,2}$ \\ ${ }^{1}$ Department of Rehabilitation Medicine, Biomedical Research Institute, Pusan National University Hospital, Busan; ${ }^{2}$ Department of Rehabilitation \\ Medicine, Pusan National University School of Medicine, Busan, Korea
}

Some patients admitted to the intensive care unit (ICU) because of an acute illness, complicated surgery, or multiple traumas develop muscle weakness affecting the limbs and respiratory muscles during acute care in the ICU. This condition is referred to as ICU-acquired weakness (ICUAW), and can be evoked by critical illness polyneuropathy (CIP), critical illness myopathy (CIM), or critical illness polyneuromyopathy (CIPNM). ICUAW is diagnosed using the Medical Research Council (MRC) sum score based on bedside manual muscle testing in cooperative patients. The MRC sum score is the sum of the strengths of the 12 regions on both sides of the upper and lower limbs. ICUAW is diagnosed when the MRC score is less than 48 points. However, some patients require electrodiagnostic studies, such as a nerve conduction study, electromyography, and direct muscle stimulation, to differentiate between CIP and CIM. Pulmonary rehabilitation in the ICU can be divided into modalities intended to remove retained airway secretions and exercise therapies intended to improve respiratory function. Physical rehabilitation, including early mobilization, positioning, and limb exercises, attenuates the weakness that occurs during critical care. To perform mobilization in mechanically ventilated patients, pretreatment by removing secretions is necessary. It is also important to increase the strength of respiratory muscles and to perform lung recruitment to improve mobilization in patients who are weaned from the ventilator. For these reasons, pulmonary rehabilitation is important in addition to physical therapy. Early recognition of CIP, CIM, and CIPNM and early rehabilitation in the ICU might improve patients' functional recovery and outcomes.

Key Words: critical illness; intensive care units; muscular diseases; physical therapy modalities; polyneuropathies; rehabilitation

\section{INTRODUCTION}

In recent years, awareness has grown regarding the importance and necessity of intensive care unit (ICU) rehabilitation, both in Korea and worldwide, and accumulating evidence has shown that ICU rehabilitation has a positive impact on patients' prognosis, quality of life, and return to normal life. Moreover, efficient ICU rehabilitation is facilitated by multidisciplinary evaluations and the establishment of systematic rehabilitation teams. As interest in ICU rehabilitation has emerged in Korea, it has been actively implemented in some leading hospitals, but the wider expansion of ICU rehabilitation to other hospitals has been hindered by a lack of awareness of its necessity, as well as concrete issues such as medical fees, facility limita-

\section{Review Article}

Received: February 9, 2019

Revised: February 19, 2019

Accepted: February 22, 2019

\section{Corresponding author}

Myung-Jun Shin

Department of Rehabilitation Medicine, Biomedical Research Institute, Pusan National University Hospital, Pusan National University School of Medicine, 179 Gudeok-ro, Seo-gu, Busan 49241, Korea

Tel: +82-51-240-7485

Fax: +82-51-247-7485

E-mail:drshinmj@gmail.com

Copyright () 2019 The Korean Society of Critical Care Medicine

This is an Open Access article distributed under the terms of Creative Attributions Non-Commercial License (http:// creativecommons.org/li-censes/by-nc/4.0/) which permits unrestricted noncommercial use, distribution, and reproduction in any medium, provided the original work is properly cited. 
tions, and staffing [1]. Thus, we conducted a literature review regarding ICU rehabilitation to assess the initial approach to ICU patients and methods of evaluating them from the perspective of rehabilitation medicine, as well as the effectiveness and practical applications of ICU rehabilitation.

\section{ICU-ACOUIRED WEAKNESS}

\section{Definition}

The occurrence of generalized muscle weakness, including weakness of the limb and respiratory muscles, during ICU admission with no causes other than acute illness is defined as ICU-acquired weakness (ICUAW) [2]. The pathogenesis of ICUAW is complex, with functional and structural involvement of the muscles and nerves. Critical illness myopathy (CIM), critical illness polyneuropathy (CIP), or both (critical illness polyneuromyopathy [CIPNM]) during the course of critical illness are the most common causes of neuromuscular weakness in ICUAW, which is frequently implicated in reduced physical function due to quadriplegia and failure to wean from the ventilator [3]. Although the onset of isolated CIP is still controversial, the incidence of CIM and CIPNM has been reported to be $25 \%-83 \%$, depending on the underlying critical illness $[4,5]$.

\section{Risk Factors}

Sepsis, systemic inflammatory response syndrome, and multiple organ failure are important risk factors for the development of ICUAW [6]. Hyperglycemia is an independent risk factor for ICUAW; thus, controlling glycemia through intensive insulin therapy and early mobilization can decrease ICUAW, which is also effective for reducing the duration of mechanical ventilation $[7,8]$. Because long-term immobilization and mechanical ventilation can cause severe limb muscle atrophy, it is necessary to reduce the duration of immobilization by ensuring early mobilization [6]. The effects of using corticosteroids and neuromuscular blocking agents in critically ill patients with ICUAW are controversial, and they are expected to affect complex pathways in ways that depend on factors such as dose, timing, glycemic control, and neuromuscular complications $[2,9]$. Age is an independent risk factor for ICUAW, and premorbid physiological muscle reserve may play an important role [2]. Decreased skeletal muscle mass at the time of ICU admission is another important risk factor for mortality and complications, and this factor merits particular consideration in elderly patients $[10,11]$. Because ICU admission occurs unexpectedly, functional assessment is limited, so the

\section{KEY MESSAGES}

- Critically ill patients experiencing weakness or difficulty weaning from the ventilator in the intensive care unit (ICU) need to be evaluated for ICU-acquired weakness (ICUAW).

- Early pulmonary and physical rehabilitation prevents ICUAW and ensures better outcomes for critically ill patients.

- A multidisciplinary approach is important, as is the role of the ICU physiatrist during ICU rehabilitation.

likelihood of ICUAW can be predicted by sarcopenia evaluation in the early stages of treatment. Images obtained from computed tomography performed at the time of admission can be referred to; alternatively, ultrasonography can be used to evaluate muscle mass at the early stage of hospitalization or to monitor muscle loss that worsens during the treatment $[12,13]$.

\section{Critical Features and Diagnosis}

In patients with CIP, distal axonal sensory-motor polyneuropathy develops in the limbs and respiratory muscles, while the facial muscles are preserved. The involvement of the limbs is symmetrical on both sides, and CIP is characterized by more severe weakness in the lower limbs and more severe distal weakness than proximal weakness, accompanied by sensory abnormalities [6]. In patients with CIM, proximal weakness is more severe than distal weakness, sensory abnormalities are preserved, muscle atrophy depends on the duration of illness, and facial weakness occurs rarely. In both conditions, muscle stretch reflexes are preserved in the early stage, but diminish as weakness progresses [3]. The diagnosis of ICUAW is confirmed via clinical features, and predisposing factors based on a physical examination and causes other than critical illness should be excluded. Performing an accurate physical examination is difficult, and in patients showing slow recovery after severe muscle weakness, an electrodiagnostic evaluation can be considered [6].

\section{Manual muscle testing}

A clinical diagnosis of ICUAW can be made through a bedside evaluation of muscle strength. The Medical Research Council (MRC) scale is used for manual muscle testing. It assesses the strength of the muscle groups of the upper and lower limbs, and an MRC sum score of less than 48 out of 60 points indicates an ICUAW diagnosis [4]. However, the MRC scale has the disadvantages of poor discrimination and a ceiling effect, 
and it is limited in that it does not distinguish between CIP and CIM. Among other methods of muscle testing, the use of handheld dynamometry and handgrip dynamometry can be considered (Figure 1). Both methods are good to excellent in terms of interrater reliability and must be applied in patients who are at least grade 3 on the MRC scale $[14,15]$. However, there is a lack of evidence regarding the diagnostic criteria of ICUAW, and more research results are needed. When evaluating critically ill patients, the cooperation, adequacy, and motivation of the patient influence the accuracy of the results, and due to restrictions in the ICU environment itself, there are limitations in accurate muscle testing. To overcome such disadvantages, strictly standardized methods should be used. Prior to a muscle strength evaluation, patients whose volitional muscle strength can be accurately evaluated should be selected based on their level of cooperation (i.e., the standardized 5 questions [S5Q] scale or the confusion assessment method for the intensive care unit) (Figure 2). In addition, it is necessary to ensure that the patient remains in a consistent position, including limb positions, joint angles, and hand position of the tester, and it is also necessary to standardize the contraction time, number of repetitions, and rest periods between tests [14]. Respiratory
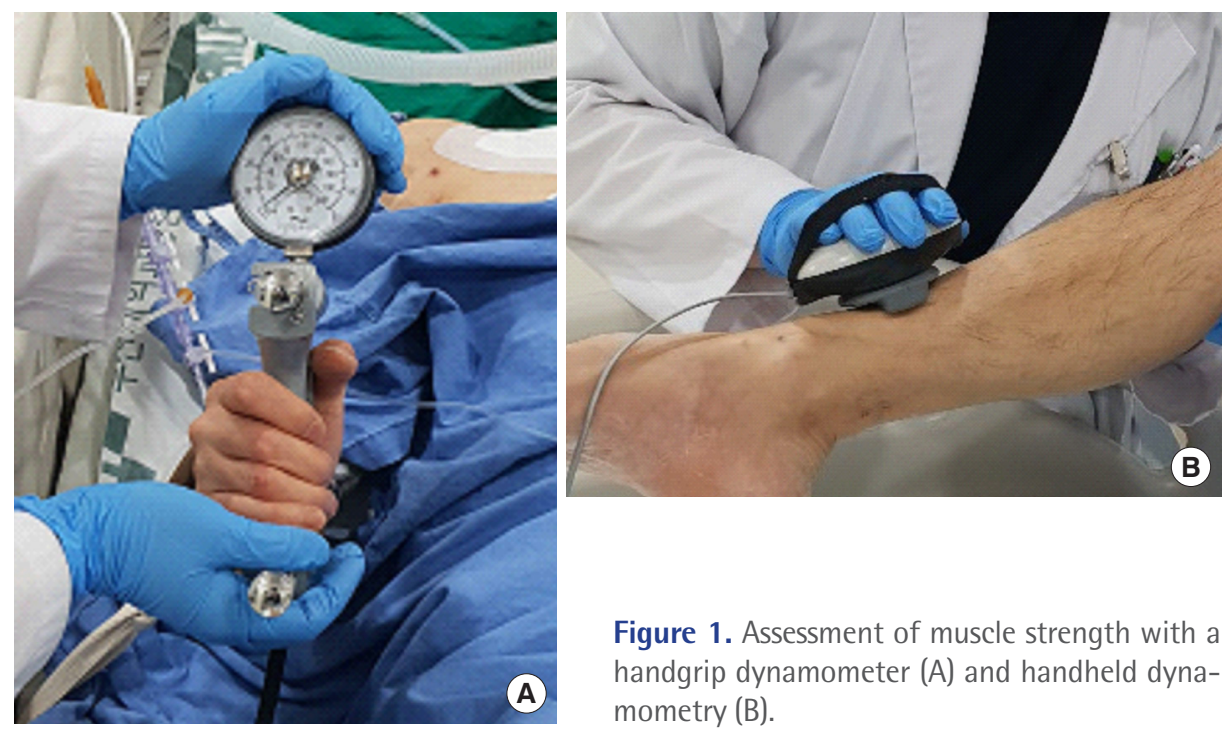

Figure 1. Assessment of muscle strength with a handgrip dynamometer $(\mathrm{A})$ and handheld dynamometry (B).

\begin{tabular}{|c|c|c|c|c|c|c|}
\hline \multicolumn{5}{|l|}{ Score 5 questions } & \multicolumn{2}{|c|}{$/ 5$} \\
\hline \multicolumn{7}{|c|}{ A. Open and close your eyes } \\
\hline \multicolumn{7}{|l|}{ B. Look at me } \\
\hline \multicolumn{7}{|c|}{ C. Open your mouth and put out your tongue } \\
\hline \multicolumn{7}{|l|}{ D. Nod your head } \\
\hline \multicolumn{7}{|c|}{ E. Raise your eyebrows when I have counted up to five } \\
\hline \multicolumn{7}{|l|}{ MRC sum score } \\
\hline & Right & Reason & EP & Left & Reason & EP \\
\hline \multicolumn{7}{|l|}{ Arm abduction } \\
\hline \multicolumn{7}{|l|}{ Forearm flexion } \\
\hline \multicolumn{7}{|l|}{ Wrist extension } \\
\hline \multicolumn{7}{|l|}{ Hip flexion } \\
\hline \multicolumn{7}{|l|}{ Knee extension } \\
\hline \multicolumn{7}{|l|}{ Ankle dorsiflexion } \\
\hline MRC sum-score & & & & 60 & & \\
\hline
\end{tabular}

Figure 2. Sample sheet used for muscle strength testing through the Medical Research Council (MRC) sum score and the standardized 5 questions (S50) at Pusan National University Hospital. For values that are difficult to assess due to peripheral or central nervous lesions, amputation, or orthopedic reasons, the values of the same muscle group on the opposite side or in proximity on the ipsilateral side are extrapolated. EP: extrapolation. 
muscle strength can also be evaluated in patients with ICUAW. Maximum inspiratory pressure can be used to assess inspiratory muscle strength, and the unidirectional valve method can be used for an accurate assessment, from which the possibility of success of weaning can be predicted [16].

In addition to direct measurements of muscle strength, ultrasound can be used to assess muscle thickness and ultrasonic echogenicity, thereby serving as a straightforward method to detect changes of muscle mass in critically ill patients [17]. Although ultrasound has limitations for diagnosing ICUAW [18], a close correlation was shown between ICU patient function and changes in the thickness of limb muscle and echogenicity; thus, ultrasound is an important and convenient tool that can be used to monitor muscle conditions during critical care [19]. Lean body mass can be easily predicted and assessed using bioelectrical impedance analysis (BIA). The phase angle $(\mathrm{PA})$ values computed using current $(\mathrm{R})$ and reactance $\left(\mathrm{X}_{\mathrm{c}}\right)$, which can be directly measured, are less affected by the hydration status of the patient than other BIA parameters [20]. The PA value signifies general cellular health and can substitute for lean body mass [21]; since low PA values are closely correlated with high mortality, PA can be used as a prognostic marker in critically ill patients. In BIA, weight is an important factor, but as there are limitations in accurately measuring patients' weight in the ICU, caution should be used when interpreting the results.

\section{Electrodiagnostic studies}

An electrodiagnostic evaluation, such as a nerve conduction study (NCS) or electromyography is necessary to diagnose CIP and CIM, and direct muscle stimulation can be additionally used to distinguish between CIP and CIM [22]. In NCSs, nerve conduction velocity is mostly normal, while the amplitude of compound muscle action potentials (CMAPs) is reduced. In patients with CIP, sensory nerve action potentials (SNAPs) are also reduced, but the test results of ICU patients who have edema should be carefully interpreted (Table 1) [2]. Because there are restrictions regarding the time and cost required to perform full electrophysiological testing in the ICU environment, the amplitudes of peroneal nerve CMAPs and sural nerve SNAPs should be selectively tested by a screening test, and additional tests can be considered in case of abnormal findings $[23,24]$.

\section{EARLY REHABILITATION IN THE ICU}

\section{Feasibility and Safety}

Due to immobilization throughout the course of critical illness, neuromuscular weakness and impairment of physical function often occur. Early rehabilitation has been proven to be safe, feasible, and important; in fact, it can be safely performed without adverse events, even when the patient uses a mechanical ventilator and undergoes continuous renal replacement therapy, extracorporeal membrane oxygenation, or femoral catheterization $[25,26]$. In addition, passive and active range of motion (ROM) exercises in neuro-ICU patients with normal or elevated intracranial pressure can be safely performed without affecting the intracranial pressure [27]. Although removal of the endotracheal tube, feeding tube, or chest tube; hemodynamic instability such as hypotension, hypertension, or desaturation; and falling can occur during rehabilitation, these events are preventable with careful patient monitoring and skilled staff who are capable of evaluating physiological changes during rehabilitation [28,29]. Prerehabilitation screening criteria to identify patients for whom rehabilitation is suitable and criteria for discontinuing rehabilitation should be established for each ICU (Table 2) [30]. Furthermore, because the culture of the ICU acts as a barrier at most centers, awareness

Table 1. Electrophysiological findings of ICUAW

\begin{tabular}{ll}
\hline Critical illness polyneuropathy & \multicolumn{1}{c}{ Critical illness myopathy } \\
\hline CMAP decreased to $<80 \%$ of LLN in $>2$ nerves & CMAP decreased to $<80 \%$ of LLN in $>2$ nerves \\
SNAP decreased to $<80 \%$ of LLN in $>2$ nerves & SNAPs are $>80 \%$ of LLN in $>2$ nerves \\
Normal conduction velocity & Normal or near-normal conduction velocity \\
Abnormal spontaneous activity & Variable spontaneous activity \\
Large polyphasic MUAPs & Small polyphasic MUAPs in $>2$ muscle groups \\
Reduced recruitment/interference pattern & Low-amplitude, full interference pattern \\
& Muscle inexcitability with direct muscle stimulation \\
\hline
\end{tabular}

ICUAW: intensive care unit-acquired weakness; CMAP: compound muscle action potential; LLN: lower limit of normal; SNAP: sensory nerve action potential; MUAP: motor-unit action potential. 
Table 2. Examples of safety criteria for starting and stopping rehabilitation in the intensive care unit

\begin{tabular}{|c|c|c|}
\hline System & Starting criteria & Stopping criteria \\
\hline \multirow[t]{3}{*}{ Cardiovascular } & Heart rate: $60-130$ beats/min & Heart rate: $<60$ or $>130$ beats/min \\
\hline & Systolic blood pressure: $90-180 \mathrm{mmHg}$, or & Systolic blood pressure: $<90$ or $>180 \mathrm{mmHg}$, or \\
\hline & mean arterial pressure: 60-100 mmHg & mean arterial pressure: $<60$ or $>100 \mathrm{mmHg}$ \\
\hline \multirow[t]{5}{*}{ Respiratory } & Respiratory rate: $5-40$ breaths/min & Respiratory rate: $<5$ or $>40$ breaths/min \\
\hline & $\mathrm{SpO}_{2} \geq 88 \%$ & $\mathrm{SpO}_{2}<88 \%$ \\
\hline & $\mathrm{FiO}_{2}<0.6$ & Concerns about the disconnection of the artificial airway \\
\hline & PEEP $<10$ & \\
\hline & Artificial airway is properly secured & \\
\hline \multirow[t]{7}{*}{ Other } & Able to open eyes to voice & Changes in consciousness \\
\hline & & New/symptomatic arrhythmia \\
\hline & & Chest pain \\
\hline & & Ventilator asynchrony \\
\hline & & Falling down \\
\hline & & Medical device removal \\
\hline & & Patient intolerance or refusal \\
\hline
\end{tabular}

$\mathrm{SpO}_{2}$ : peripheral capillary oxygen saturation; $\mathrm{FiO}_{2}$ : fraction of inspired oxygen; PEEP: positive expiratory end pressure.

of the importance of early mobilization and rehabilitation and a structured multidisciplinary effort based on smooth communication are necessary [1].

\section{Efficacy and Benefits}

Evidence for the effects of active mobilization and rehabilitation in the ICU continues to be accumulated. It is known that ICU rehabilitation has a positive effect on patients' prognosis, as assessed by improvements in functional status such as exercise capacity, muscle strength, and walking ability at discharge, as well as reductions in the duration of mechanical ventilation, length of the ICU stay, and length of hospital stay [31-34]. Furthermore, conducting very early mobilization in acute stroke patients in the neuro-ICU resulted in a rapid recovery of walking ability [35]. However, the results of previous studies are not fully consistent, as various factors affect mortality and length of hospital stay and rehabilitation requirements vary across studies. Therefore, there is a continuing need for studies to determine the effects of rehabilitation based on the disease and ICU status of patients.

\section{PULMONARY REHABILITATION}

The aims of pulmonary rehabilitation in the ICU are to recover voluntary respiration of the patient by clearing airway secretions, reducing the work of breathing, improving respiratory function, and enhancing inflation of the lungs [36]. In addi-
Table 3. Goals of pulmonary rehabilitation in the intensive care unit

\begin{tabular}{l} 
Goal of pulmonary rehabilitation \\
\hline Reducing secretion retention, atelectasis, and pneumonia \\
Maintaining or recruiting lung volume \\
Optimizing ventilation and oxygenation \\
Improving compliance and ventilation/perfusion mismatch, reducing \\
work of breathing \\
Decreasing ventilator dependence and improving residual function \\
Improving respiratory muscle strength \\
Reducing postoperative complications
\end{tabular}

tion, in patients with chest trauma, normalization of abnormal chest wall movements and restoration of the ventilatory capacity of collapsed lungs help with proper sputum removal and prevent additional pulmonary complications. Finally, the purpose of pulmonary rehabilitation is to restore the patient's normal lung function (Table 3) [37,38].

\section{Exercises for Respiratory Muscle Function}

Deep breathing exercises and incentive spirometry

Deep breathing exercises introduce air into the lungs using negative pressure generated by the patient's diaphragm instead of the accessory respiratory muscles. This can restore atelectasis, improve oxygenation and lung recruitment, increase functional residual capacity and tidal volume, and potentially help clear secretions [38]. Incentive spirometry (IS) has been 
widely used to prevent pulmonary complications and to improve lung function in nonambulatory surgical patients. Deep breathing exercises can more effectively induce maximal inspiration when patients receive visual feedback (inspired flow or volume) through IS [39]. Generally, patients perform five to 10 repetitions of a sequence of performing deep breathing slowly, holding the breath for 2 to 3 seconds, and then exhaling slowly. If sputum needs to be released, it is spit out by coughing at the end of the session (Figure 3A).

\section{Respiratory muscle training}

Weakness or fatigue of respiratory muscles and the diaphragm is an important factor in patients who fail to be weaned from mechanical ventilation. Fatigue can occur when the load of the inspiratory muscles is excessively increased due to the increase of airway resistance and decrease of lung compliance, or when there is an imbalance between respiratory muscles [40]. Additionally, prolonged ventilation itself promotes atrophy of the diaphragm and leads to its functional decrease [41]. Inspiratory muscle training can improve both inspiratory and expiratory muscle strength and reduce the duration of ventilation and weaning. However, additional research is needed to confirm the effects of training on clinical outcomes, and specific guidelines for how each training method can be used to improve strength and endurance are also necessary [42]. Threshold loading is the most general method used to determine training intensity. The threshold can be determined based on the maximum respiratory pressure (MIP) measured using the ventilator or a respiratory pressure meter. Threshold loading can be set between $20 \%$ and $50 \%$ of MIP, and generally, five sets with 6-10 breaths per set are performed once or twice a day. The threshold can be gradually increased as the patient's inspiratory muscle strength improves (Figure 3B) [43].

\section{Management of Airway Secretions}

\section{Positioning and mobilization}

Positioning and mobilization enhance ventilation and ventilation/perfusion (V/Q) mismatch, thereby assisting in oxygenation [36]. In patients with unilateral lung disease, positioning the affected lung at the uppermost location increases recruitment and promotes drainage from the lung segment, so lung function and atelectasis can be improved [44]. Moreover, in ventilated patients, the functional residual capacity and oxygenation improve and work is reduced in a seated position of greater than $30^{\circ}$ [45] because the displacement of the rib cage increases, resulting in a positive effect on minute ventilation, respiratory rate, tidal volume, and inspiratory flow rate [38]. Together with other chest physiotherapy modalities, early mobilization can result in a decreased extubation failure rate and shorter durations of mechanical ventilation and ICU stay $[46,47]$ (Figure 4). Further details on mobilization are discussed below.

\section{Hyperinflation}

The aim of lung hyperinflation is to prevent pulmonary collapse or re-expansion of collapsed alveoli by improving gas exchange through promoting secretion removal and increasing lung compliance. The cardiovascular stability of the patient must be ensured before treatment because of the large tidal volume and increased intrathoracic pressure inherent to hyperinflation. The specific protocol in each ICU varies, but the basic method is to induce expiratory flow after sufficient inspiration and 2-3 seconds of inspiratory hold. To reduce the risk of barotrauma, it is necessary to use a manometer connected to a circuit. Methods include manual hyperinflation (MHI) and ventilator hyperinflation; the sputum removal effect of both methods is known to be similar, but MHI is advantageous in that the physiotherapist receives feedback through
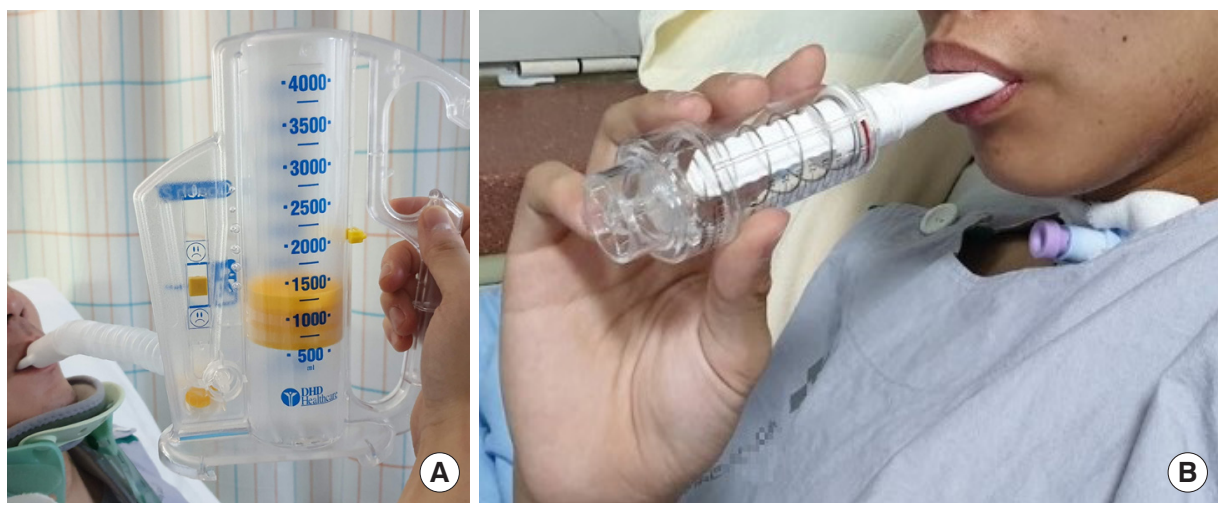

Figure 3. Incentive spirometry (A) and threshold inspiratory muscle training (B) for intensive care patients. 


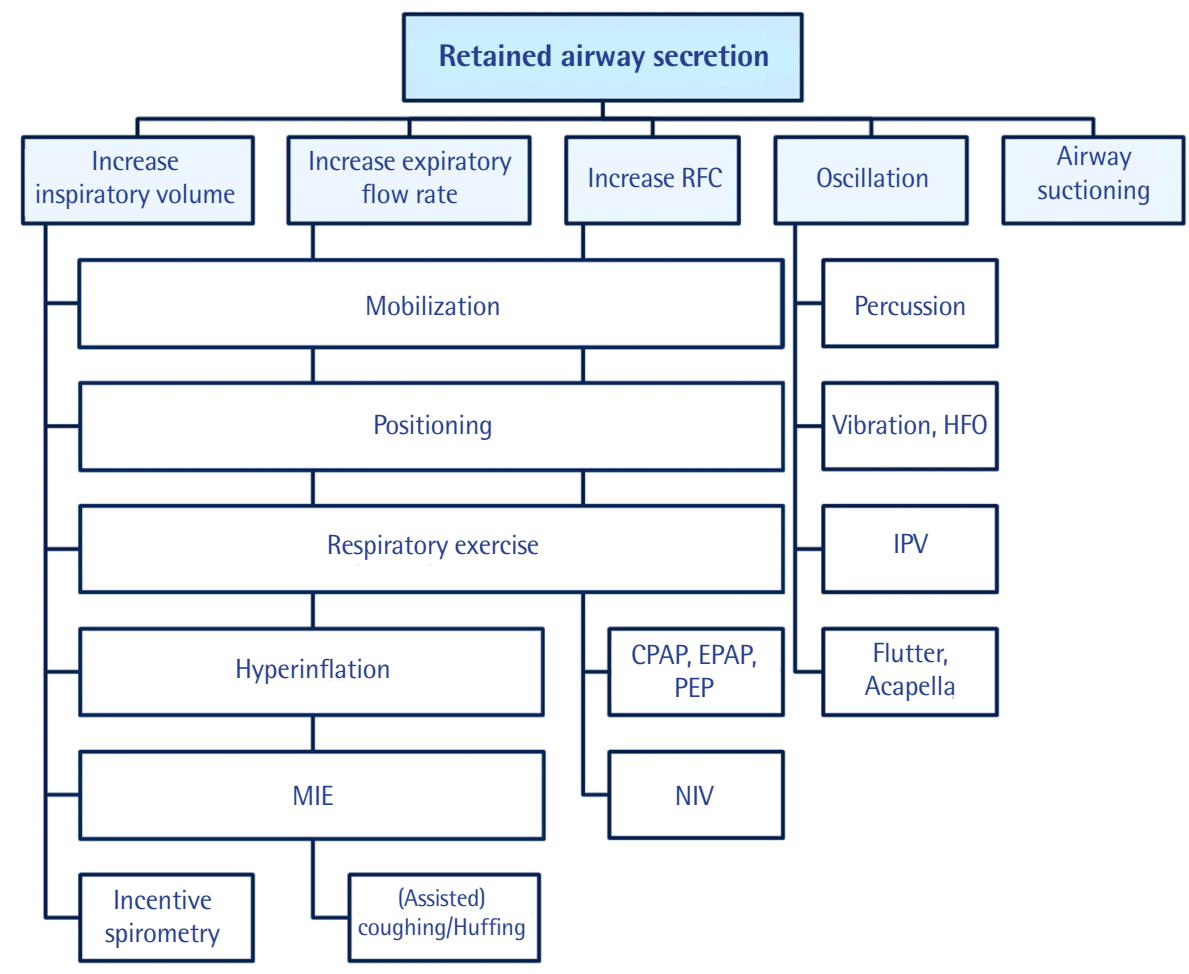

Figure 4. Treatment modalities for airway secretions. RFC: residual functional capacity; HFO: high frequency oscillation; IPV: intrapulmonary percussive ventilation; CPAP: continuous positive airway pressure; EPAP: expiratory positive airway pressure; PEP: positive expiratory pressure; NIV: noninvasive ventilation; MIE: mechanical insufflation-exsufflation.

the resuscitator bag to estimate lung compliance $[38,48]$.

\section{Mechanical insufflation-exsufflation}

Mechanical insufflation-exsufflation (MIE) is a commonly used method to remove excessive sputum from patients with neuromuscular abnormalities and is applicable to patients who do not effectively expectorate sputum due to an impaired cough. Similar to the general cough principle, the lung is inflated to a large volume with positive pressure. Subsequently, a negative pressure is quickly applied to induce sputum removal. In addition to sputum removal, MIE has the advantages of maintaining lung compliance, respiratory muscle length, and thoracic rib cage mobility. Manual techniques such as assisted cough or thoracoabdominal thrusts may be applied by the therapist along with MIE to facilitate sputum release. The use of MIE improves the likelihood of extubation in patients requiring tracheostomy and reduces the length of the postextubation ICU stay. In addition, when MIE is used in patients in whom a noninvasive ventilator (NIV) fails, it has the advantages of easier NIV adaptation and enhancement of its effects $[49,50]$. The use of MIE requires caution in patients with an undrained pneumothorax, major cardiovascular instability, or emphysematous bullae, as well as in patients with head trauma, as it may affect intracranial pressure or cerebral perfusion pressure [51].

\section{Percussion and vibrations}

In percussion, cupped hands or the palm cup is used to manually clap the affected lung and perform postural drainage. This allows shifting of secretions from the peripheral airway to the central airway, enhancing airway clearance. Vibrations can be performed manually or using mechanical devices, and conducting chest oscillation and compression together during the expiratory phase can improve peak expiratory flow rates by more than $50 \%$ (Figure $5 \mathrm{~A}$ ) [52].

\section{Oscillatory positive-expiratory-pressure devices}

Flutter and Acapella (Smiths Medical, Carlsbad, CA, USA) devices combine positive expiratory pressure therapy and highfrequency oscillations in the airway. By generating oscillations with sustained expiratory pressure, they reduce airway collapse and improve mucus discharge, enhancing lung function and oxygenation [53]. When using the Flutter device, the breath is held for 2-3 seconds after deep inspiration, and oscillations are then induced by performing slow expiration through the Flutter valve. In general, three sets of 10-15 exhalations are 

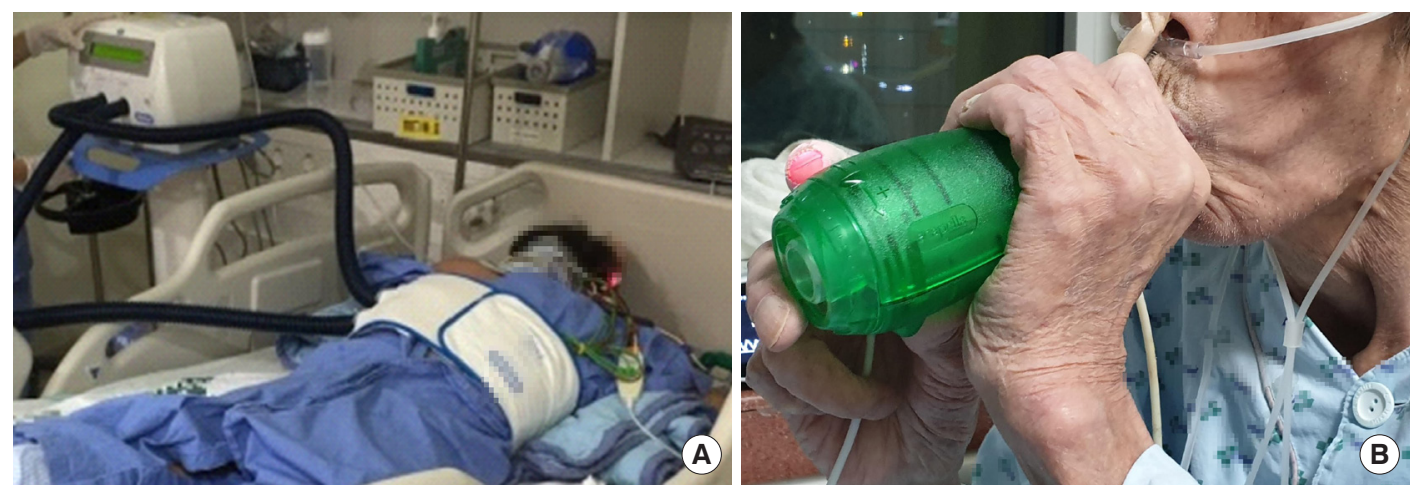

Figure 5. Postural drainage with high-frequency oscillation (A) and the Acapella device (B) for secretion removal.

performed over 12-20 minutes, and after completing each set, the "huff" cough maneuver is performed to promote sputum removal [54]. Depending on the patient's condition, this can be performed three to four times a day. The Acapella device is characterized by using a mask or mouthpiece as the interface and is applied with a nebulizer (Figure 5B).

\section{Intrapulmonary percussive ventilation}

Intrapulmonary percussive ventilation (IPV) allows simultaneous positive pressure, high-frequency oscillations, and aerosol delivery. IPV can reduce the work of breathing by increasing sputum removal and lung expansion. IPV can be effectively and safely applied in patients with cystic fibrosis, bronchiectasis, chronic obstructive pulmonary disease exacerbation, and acute respiratory failure, as well as in tracheostomized patients [55]. Generally, the treatment time is short (less than 15 minutes), and IPV can be repeated several times a day. Similar to MIE, cardiac output can decrease due to an increase of intrathoracic pressure, and adverse effects such as barotrauma and volutrauma should be considered.

\section{Chest Physiotherapy in Chest Trauma Patients}

In patients with chest trauma such as rib fractures and pulmonary contusions, the effort of inspiration increases and maximal cough strength decreases, thereby reducing lung volume and promoting atelectasis. In addition, the increased immobility period and the medications prescribed due to trauma depress the respiratory drive and hinder the recovery of lung function. For such reasons, the risk of pulmonary complications after trauma is especially high [37].

IS has not been found to have a positive effect on preventing pulmonary complications or shortening the length of hospital stay, but the incidence of acute respiratory failure may be high if the vital capacity measured by IS at the time of admission is low $[56,57]$. The use of IS starting with the initial stage of trauma allows monitoring of the patient's lung volume changes, and a sudden decrease in inspiratory volume during treatment suggests the possibility of atelectasis, pneumonia, or other pulmonary complications. The active cycle of breathing technique (ACBT) is a breathing method that helps air clearance by assisting lung ventilation and prevents infection; ACBT involves breathing control and a forced expiration technique that utilizes thoracic expansion, deep breathing, and huffing. The addition of ACBT to conventional pulmonary rehabilitation showed a significant effect on pain reduction after chest trauma [58].

\section{PHYSICAL ACTIVITY AND EARLY MOBILIZATION}

Physical activity and exercise in the ICU should be of appropriate intensity and type according to the patient's condition. Thus, accurate evaluation of the patient's cooperation level, muscle strength, joint mobility, functional status, and cardiopulmonary reserve should precede exercises, and the rehabilitation goal should be determined based on this assessment [59]. Guidelines are not defined for evaluation tools or goals, and the appropriate activity stage is determined by stepping up or down according to the guidelines of each hospital or ICU [60].

\section{Uncooperative Patient}

Positioning

Basic positioning (i.e., lateral rotational therapy) can be performed to prevent soft tissue contracture, joint contracture, peripheral nerve compression, and pressure ulcerations. The upright position can be used to increase lung volume and further improve gas exchange, although caution is required to prevent adverse effects to the cardiopulmonary system. To safely change the position of sedated patients or heavy care patients, lift use can be considered [59]. Although there is a 


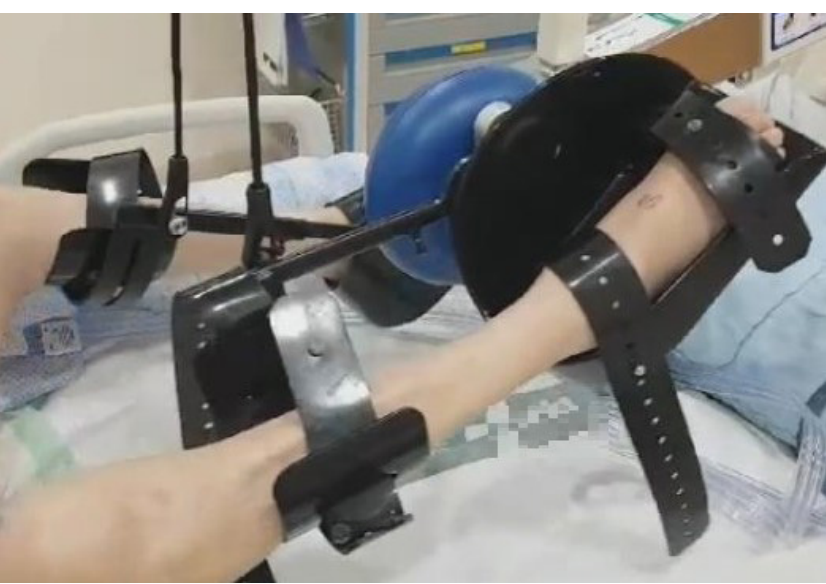

Figure 6. Cycle ergometer for active and passive cycling in the intensive care unit.

lack of evidence for the interval of position change, it is generally performed at 2 - to 4 -hour intervals, which can help reduce the incidence of pulmonary complications such as nosocomial pneumonia and atelectasis [61].

\section{Passive ROM and stretching}

Passive ROM or stretching exercises are an important treatment method to maintain the ROM and soft tissue length in patients incapable of voluntary movement. By using a continuous passive motion device, it is possible to better prevent contracture and preserve the architecture of muscle fibers [62]. Moreover, patients with severe burns, trauma, and those with central nerve damage are at a high risk for soft tissue contracture, so an additional orthosis, such as an ankle-foot orthosis, can be used to prevent joint contracture and reduce muscle tone [36].

\section{Cycle ergometer}

With technological advancements, passive cycle ergometers can also be used in sedated, immobile, and bedridden patients, which can help maintain ROM. Even after the patient recovers consciousness, active-assisted and active-resistive modes can be used to perform muscle strengthening exercises in the lower limbs, and the bedside ergometer can be used to improve quadriceps force and increase exercise capacity after ICU discharge (Figure 6) [32].

\section{Neuromuscular electrical stimulation}

Few treatment options exist for preventing the progression of muscle atrophy during prolonged immobilization periods. Neuromuscular electrical stimulation (NMES) is advantageous because it can be applied irrespective of the patient's level of
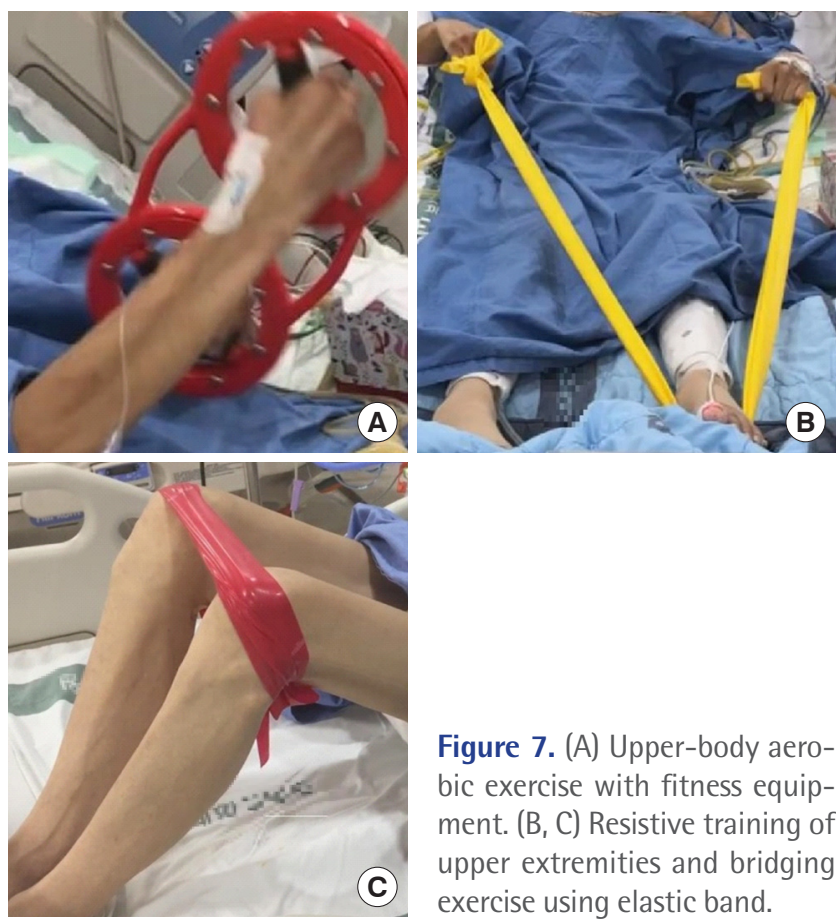

Figure 7. (A) Upper-body aerobic exercise with fitness equipment. $(B, C)$ Resistive training of upper extremities and bridging exercise using elastic band.

interaction or posture. The use of NMES promotes muscular microcirculation, delays muscle atrophy during the immobility period, and improves muscle strength and endurance $[63,64]$. NMES can be applied together with bed leg cycling, but the functional results of patients after discharge remains controversial [65]. In addition, it is necessary to establish a consensus for appropriate protocols to determine the optimal current, contraction characteristics, and targeted muscle.

\section{Tilt table treatment}

A tilt table provides partial weight bearing, which can assist in the gradual transition from bed rest to bearing the full body weight [29]. Although the evidence level for tilt table intervention is not high, it can help improve lower limb strength, prevent ankle joint contracture, and enhance patient arousal. In the beginning, only tilting without an arm support can provide effective exercise [66,67].

\section{Cooperative Patient}

Mobilization

If the patient's response to external stimulation is appropriate and cooperation is good (i.e., $S 5 \mathrm{Q} \geq 3$ ), then transitioning from passive exercise to active exercise can be attempted [68]. Mobilization generally includes sitting on the edge of the bed, moving from the bed to the chair, standing next to the bed, walking on the spot, and walking with or without ambulatory assisting devices. Particularly, during the standing and walking stages, 
caution should be taken to prevent monitoring lines, catheters, or the urinary bag from disconnecting [59]. Early and protocol-based mobilization is important for functional recovery and shortening the length of hospital stay, but insufficient evidence still exists for neuro-ICU patients [69].

\section{Aerobic exercises and resistive muscle training}

In addition to the mobilization techniques discussed above, aerobic exercises and strengthening exercises can be included. Endurance training using a bed cycle ergometer is common, and in patients with limited movement due to multiple fractures, such as lower limb fractures, an upper body ergometer can be used (Figure 7A). Resistive muscle training increases muscle mass and force generation. For an exercise effect, three sets of eight to ten repetitions with an intensity of 50\%$70 \%$ of one repetition maximum within the patient's tolerance are performed [36]. For resistive training, exercise tools such as elastic bands and pulleys can be used to perform exercises in bed (Figure 7B, C) [59]. When using tools such as the Borg Rate of Perceived Exertion, the patient's perceived fatigue can be assessed before, during, and after exercise to monitor the patient's exercise intensity [68].

\section{Critically III Trauma Patients}

Early mobilization in trauma patients has many limitations due to specific factors related to injuries and their treatment, and less evidence exists for functional outcomes. However, since the risks of prolonged immobilization and bed rest are evident, the effects of early mobilization may be enhanced when polytrauma patients are assessed jointly with the involved trauma specialists to ensure safe exercise [70]. In addition, early goal-directed mobilization in trauma patients improves mobilization during hospitalization, reduces the length of the ICU stay, and assists in enhancing functional mobility after discharge [71].

\section{CONCLUSIONS}

The safety, feasibility, and treatment effects of ICU rehabilitation in critically ill patients are well proven. The most important factor in ICU rehabilitation is to increase therapeutic effects through a multidisciplinary approach in which the treatment goals are planned and shared. The rehabilitation team should consist of an ICU physiatrist, physiotherapist, respiratory therapist, and occupational therapist. Additionally, the physiatrist should lead the team by focusing on the patient's functional evaluation and rehabilitation plan. To make ICU rehabilitation more widespread in Korea, it is necessary to address practical issues, such as the absence of an ICU rehabilitation fee and the lack of medical staff.

\section{CONFLICT OF INTEREST}

No potential conflict of interest relevant to this article was reported.

\section{ACKNOWLEDGMENTS}

This work was supported by clinical research grant from $\mathrm{Pu}$ san National University Hospital in 2019, Busan, Korea.

The patients provided written informed consent for the publication and the use of their images.

\section{ORCID}

Myung Hun Jang ～https://orcid.org/0000-0002-9694-5409

Myung-Jun Shin https://orcid.org/0000-0003-4010-0383

Yong Beom Shin https://orcid.org/0000-0001-5026-1696

\section{AUTHOR CONTRIBUTIONS}

Conceptualization: MJS. Data curation: MHJ. Formal analysis: YBS. Funding acquisition: MJS. Methodology: YBS. Project administration: YBS. Visualization: MHJ. Writing - original draft: MHJ. Writing - review \& editing: MHJ.

\section{REFERENCES}

1. Hashem MD, Parker AM, Needham DM. Early mobilization and rehabilitation of patients who are critically ill. Chest 2016; 150:722-31.

2. Hermans G, Van den Berghe G. Clinical review: intensive care unit acquired weakness. Crit Care 2015;19:274.

3. Shepherd S, Batra A, Lerner DP. Review of critical illness myopathy and neuropathy. Neurohospitalist 2017;7:41-8.

4. De Jonghe B, Sharshar T, Lefaucheur JP, Authier FJ, DurandZaleski I, Boussarsar M, et al. Paresis acquired in the intensive care unit: a prospective multicenter study. JAMA 2002;288: 2859-67.

5. Linos K, Foot C, Ziegenfuss M, Freeman WD, Tan KM. Critical illness weakness: common questions. Curr Anaesth Crit Care 2007;18:252-60

6. Latronico N, Bolton CF. Critical illness polyneuropathy and myopathy: a major cause of muscle weakness and paralysis. 
Lancet Neurol 2011;10:931-41.

7. Hermans G, De Jonghe B, Bruyninckx F, Van den Berghe G. Interventions for preventing critical illness polyneuropathy and critical illness myopathy. Cochrane Database Syst Rev 2009;(1):CD006832.

8. Patel BK, Pohlman AS, Hall JB, Kress JP. Impact of early mobilization on glycemic control and ICU-acquired weakness in critically ill patients who are mechanically ventilated. Chest 2014;146:583-9.

9. Hermans G, Wilmer A, Meersseman W, Milants I, Wouters PJ, Bobbaers H, et al. Impact of intensive insulin therapy on neuromuscular complications and ventilator dependency in the medical intensive care unit. Am J Respir Crit Care Med 2007; 175:480-9.

10. Shibahashi K, Sugiyama K, Kashiura M, Hamabe Y. Decreasing skeletal muscle as a risk factor for mortality in elderly patients with sepsis: a retrospective cohort study. J Intensive Care 2017;5:8

11. Wallace JD, Calvo RY, Lewis PR, Brill JB, Shackford SR, Sise MJ, et al. Sarcopenia as a predictor of mortality in elderly blunt trauma patients: comparing the masseter to the psoas using computed tomography. J Trauma Acute Care Surg 2017;82: 65-72.

12. Looijaard WG, Dekker IM, Stapel SN, Girbes AR, Twisk JW, Oudemans-van Straaten HM, et al. Skeletal muscle quality as assessed by CT-derived skeletal muscle density is associated with 6-month mortality in mechanically ventilated critically ill patients. Crit Care 2016;20:386.

13. Paris M, Mourtzakis M. Assessment of skeletal muscle mass in critically ill patients: considerations for the utility of computed tomography imaging and ultrasonography. Curr Opin Clin Nutr Metab Care 2016;19:125-30.

14. Vanpee G, Hermans G, Segers J, Gosselink R. Assessment of limb muscle strength in critically ill patients: a systematic review. Crit Care Med 2014;42:701-11.

15. Vanpee G, Segers J, Van Mechelen H, Wouters P, Van den Berghe G, Hermans G, et al. The interobserver agreement of handheld dynamometry for muscle strength assessment in critically ill patients. Crit Care Med 2011;39:1929-34.

16. Tzanis G, Vasileiadis I, Zervakis D, Karatzanos E, Dimopoulos S, Pitsolis T, et al. Maximum inspiratory pressure, a surrogate parameter for the assessment of ICU-acquired weakness. BMC Anesthesiol 2011;11:14.

17. Gruther W, Benesch T, Zorn C, Paternostro-Sluga T, Quittan $\mathrm{M}$, Fialka-Moser V, et al. Muscle wasting in intensive care patients: ultrasound observation of the M. quadriceps femoris muscle layer. J Rehabil Med 2008;40:185-9.
18. Witteveen E, Sommers J, Wieske L, Doorduin J, van Alfen N, Schultz MJ, et al. Diagnostic accuracy of quantitative neuromuscular ultrasound for the diagnosis of intensive care unitacquired weakness: a cross-sectional observational study. Ann Intensive Care 2017;7:40.

19. Parry SM, El-Ansary D, Cartwright MS, Sarwal A, Berney S, Koopman R, et al. Ultrasonography in the intensive care setting can be used to detect changes in the quality and quantity of muscle and is related to muscle strength and function. J Crit Care 2015;30:1151.

20. Looijaard WG, Molinger J, Weijs PJ. Measuring and monitoring lean body mass in critical illness. Curr Opin Crit Care 2018; 24:241-7.

21. Kuchnia A, Earthman C, Teigen L, Cole A, Mourtzakis M, Paris $\mathrm{M}$, et al. Evaluation of bioelectrical impedance analysis in critically ill patients: results of a multicenter prospective study. JPEN J Parenter Enteral Nutr 2017;41:1131-8.

22. Latronico N. Critical illness polyneuropathy and myopathy 20 years later. No man's land? No, it is our land! Intensive Care Med 2016;42:1790-3.

23. Moss M, Yang M, Macht M, Sottile P, Gray L, McNulty M, et al. Screening for critical illness polyneuromyopathy with single nerve conduction studies. Intensive Care Med 2014;40:68390

24. Latronico N, Nattino G, Guarneri B, Fagoni N, Amantini A, Bertolini $\mathrm{G}$, et al. Validation of the peroneal nerve test to diagnose critical illness polyneuropathy and myopathy in the intensive care unit: the multicenter Italian CRIMYNE-2 diagnostic accuracy study. F1000Res 2014;3:127.

25. Abrams D, Javidfar J, Farrand E, Mongero LB, Agerstrand CL, Ryan P, et al. Early mobilization of patients receiving extracorporeal membrane oxygenation: a retrospective cohort study. Crit Care 2014;18:R38.

26. Damluji A, Zanni JM, Mantheiy E, Colantuoni E, Kho ME, Needham DM. Safety and feasibility of femoral catheters during physical rehabilitation in the intensive care unit. J Crit Care 2013;28:535.

27. Brimioulle S, Moraine JJ, Norrenberg D, Kahn RJ. Effects of positioning and exercise on intracranial pressure in a neurosurgical intensive care unit. Phys Ther 1997;77:1682-9.

28. Nydahl P, Sricharoenchai T, Chandra S, Kundt FS, Huang M, Fischill M, et al. Safety of patient mobilization and rehabilitation in the intensive care unit: systematic review with metaanalysis. Ann Am Thorac Soc 2017;14:766-77.

29. Mendez-Tellez PA, Nusr R, Feldman D, Needham DM. Early physical rehabilitation in the ICU: a review for the neurohospitalist. Neurohospitalist 2012;2:96-105. 
30. Devlin JW, Skrobik Y, Gélinas C, Needham DM, Slooter AJ, Pandharipande PP, et al. Clinical practice guidelines for the prevention and management of pain, agitation/sedation, delirium, immobility, and sleep disruption in adult patients in the ICU. Crit Care Med 2018;46:e825-73.

31. Mendez-Tellez PA, Needham DM. Early physical rehabilitation in the ICU and ventilator liberation. Respir Care 2012;57:1663-9.

32. Burtin C, Clerckx B, Robbeets C, Ferdinande P, Langer D, Troosters T, et al. Early exercise in critically ill patients enhances short-term functional recovery. Crit Care Med 2009;37:2499505.

33. Chiang LL, Wang LY, Wu CP, Wu HD, Wu YT. Effects of physical training on functional status in patients with prolonged mechanical ventilation. Phys Ther 2006;86:1271-81.

34. Tipping CJ, Harrold M, Holland A, Romero L, Nisbet T, Hodgson CL. The effects of active mobilisation and rehabilitation in ICU on mortality and function: a systematic review. Intensive Care Med 2017;43:171-83.

35. Cumming TB, Thrift AG, Collier JM, Churilov L, Dewey HM, Donnan GA, et al. Very early mobilization after stroke fast-tracks return to walking: further results from the phase II AVERT randomized controlled trial. Stroke 2011;42:153-8.

36. Gosselink R, Bott J, Johnson M, Dean E, Nava S, Norrenberg M, et al. Physiotherapy for adult patients with critical illness: recommendations of the European Respiratory Society and European Society of Intensive Care Medicine Task Force on physiotherapy for critically ill patients. Intensive Care Med 2008;34:1188-99.

37. Nyland BA, Spilman SK, Halub ME, Lamb KD, Jackson JA, Oetting TW, et al. A preventative respiratory protocol to identify trauma subjects at risk for respiratory compromise on a general in-patient ward. Respir Care 2016;61:1580-7.

38. Pathmanathan N, Beaumont N, Gratrix A. Respiratory physiotherapy in the critical care unit. BJA Educ 2015;15:20-5.

39. Bartlett RH, Gazzaniga AB, Geraghty TR. Respiratory maneuvers to prevent postoperative pulmonary complications: a critical review. JAMA 1973;224:1017-21.

40. Moodie LH, Reeve JC, Vermeulen N, Elkins MR. Inspiratory muscle training to facilitate weaning from mechanical ventilation: protocol for a systematic review. BMC Res Notes 2011; 4:283.

41. Levine S, Nguyen T, Taylor N, Friscia ME, Budak MT, Rothenberg $\mathrm{P}$, et al. Rapid disuse atrophy of diaphragm fibers in mechanically ventilated humans. N Engl J Med 2008;358:132735.

42. Vorona S, Sabatini U, Al-Maqbali S, Bertoni M, Dres M, Bissett $\mathrm{B}$, et al. Inspiratory muscle rehabilitation in critically ill adults: a systematic review and meta-analysis. Ann Am Thorac Soc 2018;15:735-44

43. Bissett B, Leditschke IA, Green M, Marzano V, Collins S, Van Haren F. Inspiratory muscle training for intensive care patients: a multidisciplinary practical guide for clinicians. Aust Crit Care 2018 Jul 11 [Epub]. https://doi.org/10.1016/j.aucc.2018.06.001.

44. Gillespie DJ, Rehder K. Body position and ventilation-perfusion relationships in unilateral pulmonary disease. Chest 1987; 91:75-9.

45. Mezidi M, Guérin C. Effects of patient positioning on respiratory mechanics in mechanically ventilated ICU patients. Ann Transl Med 2018;6:384.

46. Lai CC, Chou W, Chan KS, Cheng KC, Yuan KS, Chao CM, et al. Early mobilization reduces duration of mechanical ventilation and intensive care unit stay in patients with acute respiratory failure. Arch Phys Med Rehabil 2017;98:931-9.

47. Wang TH, Wu CP, Wang LY. Chest physiotherapy with early mobilization may improve extubation outcome in critically ill patients in the intensive care units. Clin Respir J 2018;12:261321.

48. Ambrosino N, Venturelli E, Vagheggini G, Clini E. Rehabilitation, weaning and physical therapy strategies in chronic critically ill patients. Eur Respir J 2012;39:487-92.

49. Bach JR, Gonçalves MR, Hamdani I, Winck JC. Extubation of patients with neuromuscular weakness: a new management paradigm. Chest 2010;137:1033-9.

50. Gonçalves MR, Honrado T, Winck JC, Paiva JA. Effects of mechanical insufflation-exsufflation in preventing respiratory failure after extubation: a randomized controlled trial. Crit Care 2012;16:R48.

51. Homnick DN. Mechanical insufflation-exsufflation for airway mucus clearance. Respir Care 2007;52:1296-305.

52. McCarren B, Alison JA, Herbert RD. Manual vibration increases expiratory flow rate via increased intrapleural pressure in healthy adults: an experimental study. Aust J Physiother 2006; 52:267-71.

53. Hristara-Papadopoulou A, Tsanakas J, Diomou G, Papadopoulou O. Current devices of respiratory physiotherapy. Hippokratia 2008;12:211-20.

54. AARC clinical practice guideline. Use of positive airway pressure adjuncts to bronchial hygiene therapy: American Association for Respiratory Care. Respir Care 1993;38:516-21.

55. Paneroni M, Clini E, Simonelli C, Bianchi L, Degli Antoni F, Vitacca M. Safety and efficacy of short-term intrapulmonary percussive ventilation in patients with bronchiectasis. Respir Care 2011;56:984-8.

56. Butts CA, Brady JJ 3rd, Wilhelm S, Castor L, Sherwood A, Mc- 
Call A, et al. Do simple beside lung function tests predict morbidity after rib fractures? Am J Surg 2017;213:473-7.

57. Meynaerts A, Michels A, Leysen R, Heremans A, Sabbe M, Delooz $\mathrm{H}$, et al. The effectiveness of incentive spirometry in hospitalised patients with blunt thoracic trauma. Acta Anaesthesiol Belg 1999;50:139.

58. Grammatopoulou E, Belimpasaki V, Valalas A, Michos P, Skordilis E, Koutsouki D. Active cycle of breathing techniques contributes to pain reduction in patients with rib fractures. Hell Cheirourgike 2010;82:52-8.

59. Gosselink R, Clerckx B, Robbeets C, Vanhullebusch T, Vanpee G, Segers J. Physiotherapy in the Intensive Care Unit. Neth J Crit Care 2011;15:66-75.

60. Morris PE, Goad A, Thompson C, Taylor K, Harry B, Passmore L, et al. Early intensive care unit mobility therapy in the treatment of acute respiratory failure. Crit Care Med 2008;36:223843.

61. Schieren M, Piekarski F, Dusse F, Marcus H, Poels M, Wappler F, et al. Continuous lateral rotational therapy in trauma: a systematic review and meta-analysis. J Trauma Acute Care Surg 2017;83:926-33.

62. Griffiths RD, Palmer TE, Helliwell T, MacLennan P, MacMillan RR. Effect of passive stretching on the wasting of muscle in the critically ill. Nutrition 1995;11:428-32.

63. Edwards J, McWilliams D, Thomas M, Shah S. Electrical muscle stimulation in the intensive care unit: an integrative review. J Intensive Care Soc 2014;15:142-9.

64. Gerovasili V, Tripodaki E, Karatzanos E, Pitsolis T, Markaki V,
Zervakis D, et al. Short-term systemic effect of electrical muscle stimulation in critically ill patients. Chest 2009;136:124956.

65. Fossat G, Baudin F, Courtes L, Bobet S, Dupont A, Bretagnol A, et al. Effect of in-bed leg cycling and electrical stimulation of the quadriceps on global muscle strength in critically ill adults: a randomized clinical trial. JAMA 2018;320:368-78.

66. Chang AT, Boots R, Hodges PW, Paratz J. Standing with assistance of a tilt table in intensive care: a survey of Australian physiotherapy practice. Aust J Physiother 2004;50:51-4.

67. Bourdin G, Barbier J, Burle JF, Durante G, Passant S, Vincent $B$, et al. The feasibility of early physical activity in intensive care unit patients: a prospective observational one-center study. Respir Care 2010;55:400-7.

68. Sommers J, Engelbert RH, Dettling-Ihnenfeldt D, Gosselink $\mathrm{R}$, Spronk PE, Nollet F, et al. Physiotherapy in the intensive care unit: an evidence-based, expert driven, practical statement and rehabilitation recommendations. Clin Rehabil 2015; 29:1051-63.

69. Fuest K, Schaller SJ. Recent evidence on early mobilization in critical-Ill patients. Curr Opin Anaesthesiol 2018;31:144-50.

70. Engels PT, Beckett AN, Rubenfeld GD, Kreder H, Finkelstein JA, da Costa L, et al. Physical rehabilitation of the critically ill trauma patient in the ICU. Crit Care Med 2013;41:1790-801.

71. Schaller SJ, Anstey M, Blobner M, Edrich T, Grabitz SD, Gradwohl-Matis I, et al. Early, goal-directed mobilisation in the surgical intensive care unit: a randomised controlled trial. Lancet 2016;388:1377-88. 